OPEN ACCESS

Edited by:

Khuong Van Dinh,

Nha Trang University, Vietnam

Reviewed by:

Xuan-Vy Nguyen,

Institute of Oceanography in Nha

Trang, Vietnam

Ana F. Miranda,

RMIT University, Australia

*Correspondence:

Ahmad Zaharin Aris

zaharin@upm.edu.my

Ley Juen Loo

leyjuenlooi@upm.edu.my

Specialty section:

This article was submitted to

Marine Pollution,

a section of the journal

Frontiers in Marine Science

Received: 16 October 2020

Accepted: 23 November 2020

Published: 06 January 2021

Citation:

Looi LJ, Aris AZ, Isa NM,

Yusoff FM and Haris $H$ (2021) Elemental Composition and Health

Risk Assessment of Giant Mudskipper (Periophthalmodon schlosseri) From the Intertidal Zone of the West Coast

of Peninsular Malaysia.

Front. Mar. Sci. 7:618284.

doi: 10.3389/fmars.2020.618284

\title{
Elemental Composition and Health
} Risk Assessment of Giant Mudskipper (Periophthalmodon schlosseri) From the Intertidal Zone of the West Coast of Peninsular Malaysia

Ley Juen Looi ${ }^{1 *}$, Ahmad Zaharin Aris ${ }^{1,2,3 *}$, Noorain Mohd Isa ${ }^{1}$, Fatimah Md. Yusoff ${ }^{3,4}$ and Hazzeman Haris ${ }^{5}$

${ }^{1}$ Department of Environment, Faculty of Forestry and Environment, Universiti Putra Malaysia, Serdang, Malaysia, ${ }^{2}$ Institute of Advanced Technology, Universiti Putra Malaysia, Serdang, Malaysia, ${ }^{3}$ International Institute of Aquaculture and Aquatic Sciences, Universiti Putra Malaysia, Port Dickson, Malaysia, ${ }^{4}$ Department of Aquaculture, Faculty of Agriculture, Universiti Putra Malaysia, Serdang, Malaysia, ${ }^{5}$ School of Biological Sciences, Universiti Sains Malaysia, Gelugor, Malaysia

The elemental pollution in the intertidal zone of the West Coast of Peninsular Malaysia is of concern because this place is an ultimate sink of anthropogenic contaminants. In this study, the elemental concentrations (Al, Fe, Ba, Co, Cu, Cr, Mn, Ni, Pb, Zn, As, and $\mathrm{Cd}$ ) in various tissues (muscle, gill, liver, gastrointestinal tract, and scale) of the giant mudskippers (Periophthalmodon schlosseri) from the West Coast of Peninsular Malaysia were determined by the inductively coupled plasma-mass spectrometry. The potential health risks associated with fish muscle consumption were assessed using estimated weekly intake (EWI), target hazard quotient (THQ), and target cancer risk (TR). The results showed that relatively high concentrations of Fe $(1,641.91 \pm 96.77 \mathrm{mg} / \mathrm{kg})$, $\mathrm{Ba}(7.07 \pm 0.05 \mathrm{mg} / \mathrm{kg})$, Co $(0.32 \pm 0.00 \mathrm{mg} / \mathrm{kg}), \mathrm{Mn}(36.08 \pm 0.04 \mathrm{mg} / \mathrm{kg})$, and $\mathrm{Ni}$ $(4.43 \pm 0.06 \mathrm{mg} / \mathrm{kg}$ ) were found in the fish scales, suggesting the uptake waterborne elements via dermal exposure. However, all studied elements in the fish muscle were below the national and international food permissible limits. Health risk assessment through the estimation of $\mathrm{THQ}$ suggested that long-term exposure of As through fish consumption would cause possible non-carcinogenic risk. Also, the estimation of TR indicated that consumers are susceptible to cancer risks because of $\mathrm{Cr}$ and As exposure via fish consumption. Thus, for a developing country like Malaysia, it is recommended to include continuous monitoring of intertidal zones, using potential bioindicators, to assess ecosystem health status and safeguard human health. Additionally, mitigation strategies to address estuaries contamination and efforts to conserve and sustain natural resources should be established.

Keywords: food safety, giant mudskipper, health risk assessment, target cancer risk, target hazard quotient, trace element 


\section{INTRODUCTION}

The intertidal zone is a peculiar and narrow edge of intersection area where the land and the sea meet (Hwang et al., 2019). This area has various productive ecosystems, such as estuaries, mangroves, and mudflats, that serve as habitat for numerous species of aquatic organisms (Khaironizam and Norma-Rashid, 2005). This area also plays an important role in nutrient cycling, waves and storms protection, and purification of contaminants (MacKinnon et al., 2012). Despite the quantity of ecosystem services provided by the intertidal zone, the area is facing unprecedented pressure brought by hinterland anthropogenic activities, such as industrial, agricultural, deforestation, fishing, logging, and mining, thereby affecting the aquatic organisms (Khatri and Tyagi, 2015; Madricardo et al., 2019; Veiga et al., 2019).

In recent years, environmental scientists from many countries have given much attention to this area, particularly in monitoring programs to assess elemental contamination (Wilkes et al., 2017; Gu, 2018; Hwang et al., 2019; Veiga et al., 2019). Human activities along with sea-based and land-based pollution have deteriorated the environmental quality of intertidal zones (Looi et al., 2019; Sany et al., 2019; Zhao et al., 2019). In Malaysia, much of scientific monitoring research related to elemental pollution in intertidal zones had been carried along the West Coast of Peninsular Malaysia (Yap et al., 2003a,b, 2009; Ikram et al., 2010; Yap and Pang, 2011; Haris and Aris, 2015) because the majority of industrial, agricultural, and economic activities are concentrated in this area (Shazili et al., 2006). Once trace elements are discharged to the intertidal zone from inland sources via various environmental pathways (Hwang et al., 2019), they are persistent in the environment and could bioaccumulate in aquatic organisms (Mottalib et al., 2018; Ali et al., 2019). In the intertidal zone, sediment acts as important sinks of trace elements and is able to retain contaminants from various pollution sources (Aris and Looi, 2015; Zhu et al., 2018; ThanhNho et al., 2019). Macrobenthic fauna living in close contact with intertidal sediments is especially susceptible to trace elements contamination and is able to accumulate both essential and nonessential elements (Ahmed et al., 2011; Tavakoly Sany et al., 2014; Veiga et al., 2019). Following this, benthic biota, such as oysters, clams, cockles (Hossen et al., 2015), and snails (Yap and Cheng, 2009), have been mined for relevant bioindicator species and are extensively used as biomonitoring tools in determining elemental pollution status of intertidal zones in the Malaysian coast. However, other potential bioindicators are yet to be revealed and used to reflect the health status of the aquatic system.

In fact, demersal fish could accumulate higher metal concentrations than other fish species (Naccari et al., 2015). Demersal fishes that live and feed at the bottom of the sea are sensitive to pollution and could absorb and build up toxicants in the food chain (Rajeshkumar and Li, 2018). The accumulation of excessive amount of trace elements all the way up to the food chain could pose potential health risks to humans who are at the top of the food chain (Ali et al., 2019; Liu et al., 2019). For these reasons, demersal fish could be used as a potential bioindicator for elemental contamination in intertidal coastal mudflats. Following this, numerous studies have been carried out worldwide to identify fish species that could be used as a bioindicator for elemental contamination in intertidal zones (Ikram et al., 2010; Looi et al., 2016; Ahmed et al., 2019; Trevizani et al., 2019).

The giant mudskipper (Gobiidae: Oxudercinae, Periophthalmodon schlosseri) is a goggled-eyed amphibious fish that has adapted gills that allows it to live and breed on the mudflats (Ikram et al., 2010; You et al., 2018). This air-breathing goby can be found widely along the shores of tropical and subtropical countries, such as Indonesia, India, and Malaysia, and continents, such as Africa and Australia (Omar et al., 2010). They are carnivorous that feed mainly on fiddler crabs, medaka fish, and juvenile and intermediate fishes (Zulkifli et al., 2012). Like many other fishes, besides having great value of high-quality protein, giant mudskippers do play an important role in the food chain ( $\mathrm{Ng}$ et al., 2019). To date, limited information is available concerning elemental contamination in the giant mudskipper. Few studies have been carried out to determine if the giant mudskipper is a suitable potential bioindicator species for elemental pollution in intertidal zones (Ikram et al., 2010; Buhari and Ismail, 2016; Looi et al., 2016; Ng et al., 2019). In Malaysia, Ikram et al. (2010) proposed that the giant mudskipper could be used as a potential bioindicator for copper $(\mathrm{Cu})$, lead $(\mathrm{Pb})$, and cadmium $(\mathrm{Cd})$ pollution in intertidal zones. However, the study conducted by Ikram et al. (2010) only focused on selected important elements (i.e., $\mathrm{Cd}, \mathrm{Cu}$, and $\mathrm{Pb}$ ); thus, a more comprehensive monitoring study concerning a wide range of elements is indispensable to fill in the gap of study. Such study is timely and could be complementary in providing a clearer picture of the possible application of the giant mudskipper as a bioindicator for elemental monitoring in tropical intertidal zones. This study aims to (i) determine the elemental concentrations ( $\mathrm{Al}, \mathrm{Fe}, \mathrm{Ba}, \mathrm{Co}, \mathrm{Cu}, \mathrm{Cr}, \mathrm{Mn}, \mathrm{Ni}, \mathrm{Pb}$, $\mathrm{Zn}, \mathrm{As}$, and $\mathrm{Cd}$ ) in tissues (i.e., muscle, gill, liver, gastrointestinal tract, and scale) of the giant mudskipper and (ii) assess the potential health risks associated with fish muscle consumption.

\section{MATERIALS AND METHODS}

\section{Sampling Sites}

The majority of the population and industry development are concentrated in the West Coast of Peninsular Malaysia. This area is constantly exposed to land-based and marine-based pollutants, thus affecting both ecology and human health (Sany et al., 2019). In the present study, a total of 135 giant mudskippers (P. schlosseri) ( $n=15$ for each sampling site) were collected from selected intertidal mudflats along the West Coast of Peninsular Malaysia (Figure 1) from November to December 2013.

\section{Analytical Procedure}

The total weight and length of collected samples were measured (Table 1). The collected samples were kept cold $\left(4^{\circ} \mathrm{C}\right)$ in acidwashed polyethylene zip-lock bags before being transported to the laboratory for further analysis. In the laboratory, giant mudskippers were immediately dissected into different tissues 


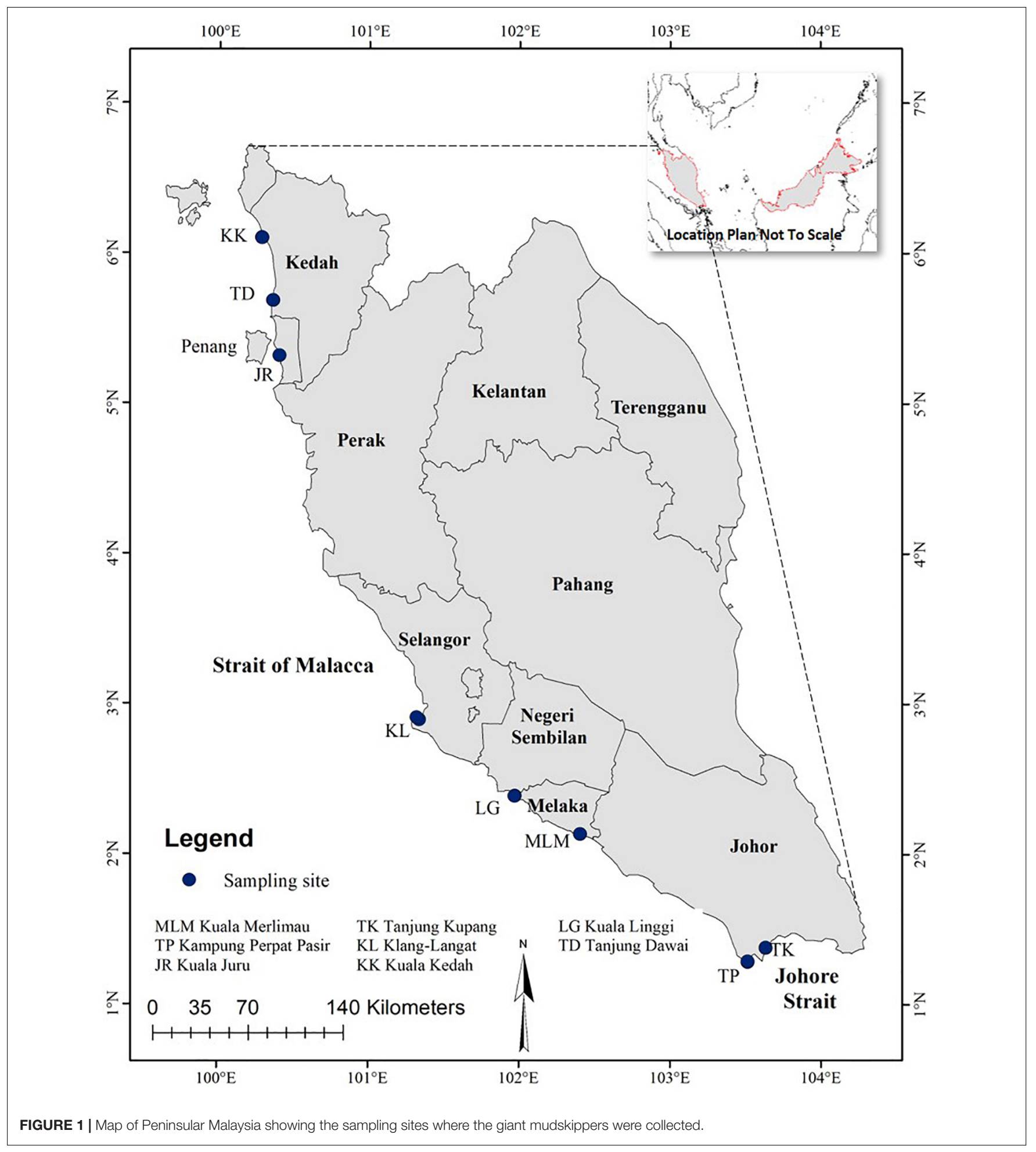

(i.e., muscle, gill, liver, gastrointestinal tract, and scale) and freeze-dried before being grinded into fine powder for acid digestion and elemental analysis. The total extractable elements (Al, Fe, Ba, Co, Cu, Cr, Mn, Ni, Pb, Zn, As, and $\mathrm{Cd}$ ) in tissues were extracted following the aqua regia method described by EPA Method 3051A (USEPA Method 3051A, 2007) and
Radojević and Bashkin (2006). Briefly, approximately $1.0 \mathrm{~g}$ of homogenized tissues was acid digested in a microwave system with $15 \mathrm{ml}$ of aqua regia solution $\left[\mathrm{HCl}: \mathrm{HNO}_{3}, 3: 1(\mathrm{v} / \mathrm{v})\right]$ at $180^{\circ} \mathrm{C}$ for $9.5 \mathrm{~min}$. The digested samples were filtered through a $0.45 \mu \mathrm{m}$ membrane filter into a $50 \mathrm{ml}$ volumetric flask and topped up to $50 \mathrm{ml}$ with $0.25 \mathrm{M} \mathrm{HNO}_{3}$ prior to elemental analysis using an 
TABLE 1 | The range of wet body weight (g), fork length (cm), and Fulton condition factor of giant mudskippers collected from the West Coast of Peninsular Malaysia.

\begin{tabular}{lcccc}
\hline Sampling site & $\boldsymbol{n}$ & Length $(\mathbf{c m})$ & Weight $(\mathbf{g})$ & $\begin{array}{c}\text { Fulton condition } \\
\text { factor }(\boldsymbol{K})\end{array}$ \\
\hline KK & 15 & $16.4-23.2$ & $42.0-121.0$ & $0.76-1.01$ \\
TD & 15 & $13.8-25.0$ & $22.0-120.0$ & $0.77-1.29$ \\
JR & 15 & $12.0-20.4$ & $8.0-82.0$ & $0.46-1.26$ \\
KL & 15 & $12.5-20.5$ & $10.0-82.0$ & $0.50-1.17$ \\
LG & 15 & $21.1-27.0$ & $80.0-82.0$ & $0.41-0.87$ \\
MLM & 15 & $19.5-25.0$ & $75.0-120.0$ & $0.51-1.13$ \\
P & 15 & $17.2-23.1$ & $45.0-120.0$ & $0.72-1.20$ \\
TP & 15 & $16.4-23.4$ & $40.0-122.0$ & $0.80-1.04$ \\
TK & 15 & $13.4-22.0$ & $20.0-100.0$ & $0.82-0.94$ \\
\hline
\end{tabular}

inductively coupled plasma-mass spectrometry (ICP-MS, ELAN DRC-e; PerkinElmer, Shelton, CT, United States).

\section{Quality Assurance and Quality Control}

All the apparatus and glassware used in this study were acidwashed with $5 \% \mathrm{HNO}_{3}$. Triplicate and homogenized samples were collected from each sampling site to account for variability. Method blanks were prepared in parallel with the samples during acid digestion. The standard and method blanks were used to define the background correction. Besides, certified reference material [DORM-4: National Research Council Canada (NRCCNRC), Canada] was used to validate the analytical method, and the recovery was found to fall within $83.55-107.70 \%$ (Table 2).

\section{Potential Human Health Risk Assessment for Fish Consumption}

Human health risk assessment is a process of estimating the nature and probability of adverse human health effects to those who may be exposed to elements or pollutants in contaminated environmental media (USEPA, 2016). Potential risk of human exposure to elements ( $\mathrm{Al}, \mathrm{Fe}, \mathrm{Ba}, \mathrm{Co}, \mathrm{Cu}, \mathrm{Cr}, \mathrm{Mn}, \mathrm{Ni}, \mathrm{Pb}$, $\mathrm{Zn}$, As, and $\mathrm{Cd}$ ) was assessed by calculating the estimated weekly intake (EWI) (Equation 1) based on the average fish consumption of the giant mudskipper at $0.03 \mathrm{~kg}$ per capita week $^{-1}$ (3.89 g/person/day) (GEMS/WHO, 2012).

Estimated Weekly Intake (EWI) $(\mu \mathrm{g})$ per kg body weight $=$

$$
\frac{\mathrm{C}_{\mathrm{fish}} \times \mathrm{IR}}{B W}
$$

where $C_{\text {fish }}$ represents the elements concentration in giant mudskippers, IR is the fish consumption rate (kg/week), and BW is the average body weight $(60 \mathrm{~kg})$. The EWIs were compared with the Provisional Tolerable Weekly Intake (PTWI) established by the Joint FAO/WHO Expert Committee on Food Additives (JECFA).

Next, the target hazard quotient (THQ) (Equation 2) was used to assess the non-cancer risk of element contamination through the consumption of giant mudskippers. The THQ for a given element is calculated as a ratio between the estimated exposure doses to the reference dose (RfD) (Ahmed et al., 2015). The
TABLE 2 | Measured and certified values of elemental concentrations ( $\mathrm{Fe}, \mathrm{Cu}, \mathrm{Cr}$, $\mathrm{Ni}, \mathrm{Pb}, \mathrm{Zn}, \mathrm{As}$, and $\mathrm{Cd}$ ) for certified reference material DORM-4 (mg/kg).

\begin{tabular}{lrrc}
\hline Elements & Certified value & Measured value & Recovery (\%) \\
\hline $\mathrm{Fe}$ & $341.00 \pm 27.00$ & $284.00 \pm 1.85$ & 83.55 \\
$\mathrm{Cu}$ & $15.90 \pm 0.90$ & $14.24 \pm 0.13$ & 89.56 \\
$\mathrm{Cr}$ & $1.87 \pm 0.16$ & $1.66 \pm 0.01$ & 88.54 \\
$\mathrm{Ni}$ & $1.36 \pm 0.22$ & $1.33 \pm 0.03$ & 97.65 \\
$\mathrm{~Pb}$ & $0.42 \pm 0.05$ & $0.39 \pm 0.00$ & 94.61 \\
$\mathrm{Zn}$ & $52.20 \pm 3.20$ & $51.39 \pm 0.49$ & 98.45 \\
$\mathrm{As}$ & $6.80 \pm 0.64$ & $7.32 \pm 0.22$ & 107.70 \\
$\mathrm{Cd}$ & $0.31 \pm 0.02$ & $0.30 \pm 0.01$ & 98.20 \\
\hline
\end{tabular}

RfDs used in this study were obtained from the online database of USEPA's Integrated Risk Information System (IRIS) summary table.

$$
T H Q=\frac{E F \times E D \times I R \times C m}{R f D \times B W \times A T} \times 10-3
$$

where EF is the exposure frequency (365 days/year), ED is the exposure duration (26 years), IR is the giant mudskipper ingestion rate $\left(3.89 \mathrm{~g} /\right.$ person day $\left.^{-1}\right), C_{\mathrm{m}}$ is the element concentration in giant mudskippers ( $\mathrm{mg} / \mathrm{kg}$ wet weight), $\mathrm{RfD}$ is the oral reference dose $\left(\mathrm{mg} / \mathrm{kg} \mathrm{day}{ }^{-1}\right)(\mathrm{As}=0.0003, \mathrm{Ba}=0.2$, $\mathrm{Cd}=0.001, \mathrm{Cr}=1.5, \mathrm{Mn}=0.14, \mathrm{Ni}=0.02$, and $\mathrm{Zn}=0.3)(\mathrm{USEPA}$, $2000)$, BW is the average body weight (60 kg), and AT is the averaging exposure time for non-carcinogens $(\mathrm{EF} \times \mathrm{ED})$. It is unlikely to experience adverse health effect if the THQ is less than 1 . Conversely, THQ exceeding 1 suggests a potential risk to human health (Gbogbo et al., 2018).

The total THQ (TTHQ) (Equation 3) was used to assess the cumulative non-carcinogenic risk of multiple elements, as the adverse effects of elements on human health are due to multiple elements. TTHQ less than or equal to 1 suggests that there are no significant non-cancer risks; TTHQ greater than 1 indicates that there are potential adverse effects on human health (Wei and Cen, 2020).

$$
T T H Q=\sum_{i=1}^{12} T H Q_{i}
$$

Meanwhile, the target cancer risk (TR) was used to estimate the lifetime cancer risk due to the consumption of giant mudskippers (Equation 4).

$$
T R=\frac{E F \times E D \times I R \times C m \times C S F}{B W \times A T} \times 10^{-3}
$$

where CFS is the oral carcinogenic slope factor $\left(\mathrm{mg} / \mathrm{kg} \mathrm{day}^{-1}\right)$ obtained from the online database of EPA's IRIS (As $=1.5$, $\mathrm{Cr}=0.5, \mathrm{~Pb}=0.0085)$. This study assumed that $10 \%$ of the total As concentration in fish is inorganic As (Gbogbo et al., 2018) to prevent overestimation of the health risk associated with dietary As exposure. According to Ahmed et al. (2015), the acceptable TR range for carcinogens is from $10^{-4}$ to $10^{-6}$. Basically, TR value $1 \times 10^{-6}$ means that there is a possibility of developing one cancer case in 1 million individuals (USEPA, 2020). 


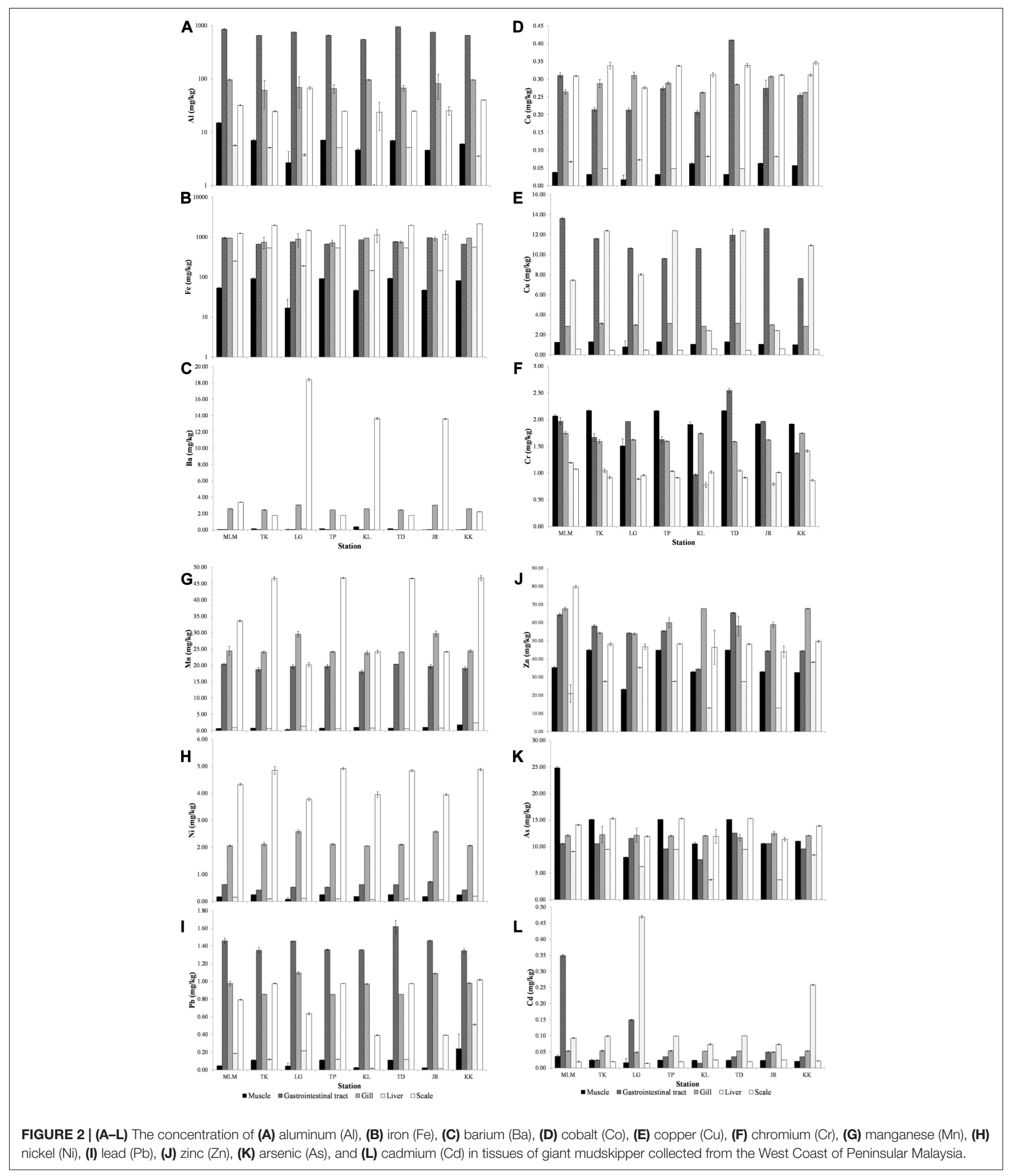

The allowable meal size that may be consumed over a period of time must be assessed when the elemental concentration of giant mudskippers is found to cause an acceptable non-cancer or cancer risk. This assessment is highly recommended as it provides valuable information on the amount of fish that can be taken and would not be expected to cause adverse health effects. The 
TABLE 3 | Comparison of elemental concentration ( $\mathrm{mg} / \mathrm{kg}$ ) in giant mudskippers from current study with previous studies and guidelines.

\begin{tabular}{|c|c|c|c|c|c|c|c|}
\hline Biota & Tissues & $\begin{array}{c}\text { Al } \\
\text { Mean } \pm \text { SD }\end{array}$ & $\begin{array}{c}\text { Fe } \\
\text { Mean } \pm \text { SD }\end{array}$ & $\begin{array}{c}\mathrm{Ba} \\
\text { Mean } \pm \text { SD }\end{array}$ & $\begin{array}{c}\text { Co } \\
\text { Mean } \pm \text { SD }\end{array}$ & Location & References \\
\hline \multirow{5}{*}{$\begin{array}{l}\text { Giant mudskipper (Periophthalmodon } \\
\text { schlosseri) }\end{array}$} & Muscle & $6.78 \pm 0.35$ & $65.39 \pm 3.06$ & $0.13 \pm 0.01$ & $0.04 \pm 0.00$ & West Coast Peninsular Malaysia & Present study \\
\hline & Gastrointestinal tract & $726.94 \pm 5.42$ & $792.31 \pm 8.20$ & $0.05 \pm 0.00$ & $0.27 \pm 0.01$ & West Coast Peninsular Malaysia & Present study \\
\hline & Gill & $79.07 \pm 17.28$ & $861.74 \pm 103.39$ & $2.65 \pm 0.01$ & $0.28 \pm 0.00$ & West Coast Peninsular Malaysia & Present study \\
\hline & Liver & $3.80 \pm 0.07$ & $364.31 \pm 1.74$ & $0.02 \pm 0.00$ & $0.10 \pm 0.00$ & West Coast Peninsular Malaysia & Present study \\
\hline & Scale & $32.90 \pm 2.89$ & $1641.91 \pm 96.77$ & $7.07 \pm 0.05$ & $0.32 \pm 0.00$ & West Coast Peninsular Malaysia & Present study \\
\hline \multirow{3}{*}{$\begin{array}{l}\text { Giant mudskipper (Periophthalmodon } \\
\text { schlosseri) }\end{array}$} & Muscle & NA & NA & NA & NA & Morib, Malaysia & Ikram et al., 2010 \\
\hline & Gill & NA & NA & NA & NA & Morib, Malaysia & Ikram et al., 2010 \\
\hline & Liver & NA & NA & NA & NA & Morib, Malaysia & Ikram et al., 2010 \\
\hline \multirow{3}{*}{$\begin{array}{l}\text { Giant mudskipper (Periophthalmodon } \\
\text { schlosseri) }\end{array}$} & Muscle & NA & NA & NA & NA & Remis, Malaysia & Ikram et al., 2010 \\
\hline & Gill & NA & NA & NA & NA & Remis, Malaysia & Ikram et al., 2010 \\
\hline & Liver & NA & NA & NA & NA & Remis, Malaysia & Ikram et al., 2010 \\
\hline \multirow{5}{*}{$\begin{array}{l}\text { Giant mudskipper (Periophthalmodon } \\
\text { schlosseri) }\end{array}$} & Muscle & NA & NA & NA & NA & Sg. Puluh, Klang, Malaysia & Buhari and Ismail T. R., 2017 \\
\hline & Intestine & NA & NA & NA & NA & Sg. Puluh, Klang, Malaysia & Buhari and Ismail T. R., 2017 \\
\hline & Gill & NA & NA & NA & NA & Sg. Puluh, Klang, Malaysia & Buhari and Ismail T. R., 2017 \\
\hline & Liver & NA & NA & NA & NA & Sg. Puluh, Klang, Malaysia & Buhari and Ismail T. R., 2017 \\
\hline & Scale & NA & NA & NA & NA & Sg. Puluh, Klang, Malaysia & Buhari and Ismail T. R., 2017 \\
\hline Mudskipper (Periophthalmus waltoni) & Muscle & NA & NA & NA & NA & Hormuz Strait, Persian Gulf & Sarhadizadeh et al., 2013 \\
\hline \multirow[t]{2}{*}{ Mudskipper (Periophthalmus waltoni) } & Muscle & NA & NA & NA & NA & Khuzestan, Iran & Sary and Mohammadi, 2012 \\
\hline & Liver & NA & NA & NA & NA & Khuzestan, Iran & Sary and Mohammadi, 2012 \\
\hline Mudskipper (Gobius boddarti) & Muscle & NA & $1051 \pm 38.42$ & NA & NA & Dublar Char, Bangladesh & Ahmed et al., 2011 \\
\hline Mudskipper (Periophthalmus barbarus) & Muscle & NA & NA & $22.00-510.00$ & NA & Port Harcourt, Nigeria & Nwakanma and Hart, 2013 \\
\hline Malaysian Food Regulation 1985 & & NA & NA & NA & NA & & Malaysian Food Act, 2004 \\
\hline $\begin{array}{l}\text { CODEX STAN 193-1995 (Amendment: } \\
\text { 2015) }\end{array}$ & & NA & NA & NA & NA & & $\begin{array}{l}\text { Codex Alimentarius } \\
\text { Commission [CAC], } 2015\end{array}$ \\
\hline $\begin{array}{l}\text { European Commission (EC) No } \\
\text { 1881/2006 }\end{array}$ & & NA & NA & NA & NA & & European Commission, 2006 \\
\hline $\begin{array}{l}\text { Australia New Zealand Food Standards } \\
\text { Code-Schedule } 19\end{array}$ & & NA & NA & NA & NA & & $\begin{array}{l}\text { Australian Government Federal } \\
\text { Register of Legislation, } 2017\end{array}$ \\
\hline $\begin{array}{l}\text { Hong Kong Cap } 132 \text { V Food } \\
\text { Adulteration (Metallic contamination) } \\
\text { Regulations }\end{array}$ & & NA & NA & NA & NA & & HK Law, 2019 \\
\hline $\begin{array}{l}\text { Singapore Food Regulations } 1998 \\
\text { (Revised: 2005) }\end{array}$ & & NA & NA & NA & NA & & $\begin{array}{l}\text { Singapore Food Regulations, } \\
2005\end{array}$ \\
\hline
\end{tabular}


TABLE 3 | Continued

\begin{tabular}{|c|c|c|c|c|c|c|c|}
\hline Biota & Tissues & $\begin{array}{c}\mathrm{Cu} \\
\text { Mean } \pm \text { SD }\end{array}$ & $\begin{array}{c}\text { Cr } \\
\text { Mean } \pm \text { SD }\end{array}$ & $\begin{array}{c}\text { Mn } \\
\text { Mean } \pm \text { SD }\end{array}$ & $\stackrel{\mathrm{Ni}}{\text { Mean } \pm \mathrm{SD}}$ & Location & References \\
\hline \multirow{5}{*}{$\begin{array}{l}\text { Giant mudskipper (Periophthalmodon } \\
\text { schlosseri) }\end{array}$} & Muscle & $1.14 \pm 0.08$ & $1.98 \pm 0.03$ & $0.92 \pm 0.04$ & $0.20 \pm 0.01$ & West Coast Peninsular Malaysia & Present study \\
\hline & Gastrointestinal tract & $11.04 \pm 0.10$ & $1.76 \pm 0.03$ & $19.45 \pm 0.47$ & $0.56 \pm 0.00$ & West Coast Peninsular Malaysia & Present study \\
\hline & Gill & $3.00 \pm 0.02$ & $1.66 \pm 0.01$ & $25.52 \pm 0.56$ & $2.21 \pm 0.03$ & West Coast Peninsular Malaysia & Present study \\
\hline & Liver & $8.55 \pm 0.04$ & $1.02 \pm 0.02$ & $1.06 \pm 0.01$ & $0.11 \pm 0.00$ & West Coast Peninsular Malaysia & Present study \\
\hline & Scale & $0.52 \pm 0.01$ & $0.96 \pm 0.01$ & $36.08 \pm 0.04$ & $4.43 \pm 0.06$ & West CoastPeninsular Malaysia & Present study \\
\hline \multirow{3}{*}{$\begin{array}{l}\text { Giant mudskipper (Periophthalmodon } \\
\text { schlosseri) }\end{array}$} & Muscle & $0.80 \pm 0.10$ & NA & NA & NA & Morib, Malaysia & Ikram et al., 2010 \\
\hline & Gill & $1.30 \pm 0.10$ & NA & NA & NA & Morib, Malaysia & Ikram et al., 2010 \\
\hline & Liver & $5.30 \pm 0.60$ & NA & NA & NA & Morib, Malaysia & Ikram et al., 2010 \\
\hline \multirow{3}{*}{$\begin{array}{l}\text { Giant mudskipper (Periophthalmodon } \\
\text { schlosseri) }\end{array}$} & Muscle & $0.90 \pm 0.03$ & NA & NA & NA & Remis, Malaysia & Ikram et al., 2010 \\
\hline & Gill & $1.60 \pm 0.10$ & NA & NA & NA & Remis, Malaysia & Ikram et al., 2010 \\
\hline & Liver & $6.60 \pm 0.10$ & NA & NA & NA & Remis, Malaysia & Ikram et al., 2010 \\
\hline \multirow{5}{*}{$\begin{array}{l}\text { Giant mudskipper (Periophthalmodon } \\
\text { schlosseri) }\end{array}$} & Muscle & $5.45 \pm 0.28^{a}$ & NA & NA & $8.74 \pm 0.72^{\mathrm{a}}$ & Sg. Puluh, Klang, Malaysia & Buhari and Ismail T. R., 2017 \\
\hline & Intestine & $15.56 \pm 0.79^{a}$ & NA & NA & $12.31 \pm 1.31^{\mathrm{a}}$ & Sg. Puluh, Klang, Malaysia & Buhari and Ismail T. R., 2017 \\
\hline & Gill & $7.83 \pm 0.37^{a}$ & NA & NA & $10.87 \pm 0.84^{a}$ & Sg. Puluh, Klang, Malaysia & Buhari and Ismail T. R., 2017 \\
\hline & Liver & $14.67 \pm 1.18^{\mathrm{a}}$ & NA & NA & $12.51 \pm 0.85^{a}$ & Sg. Puluh, Klang, Malaysia & Buhari and Ismail T. R., 2017 \\
\hline & Scale & $7.09 \pm 0.03^{a}$ & NA & NA & $12.35 \pm 2.12^{\mathrm{a}}$ & Sg. Puluh, Klang, Malaysia & Buhari and Ismail T. R., 2017 \\
\hline Mudskipper (Periophthalmus waltoni) & Muscle & NA & NA & NA & $0.35-3.15$ & Hormuz Strait, Persian Gulf & Sarhadizadeh et al., 2013 \\
\hline \multirow[t]{2}{*}{ Mudskipper (Periophthalmus waltoni) } & Muscle & NA & NA & NA & NA & Khuzestan, Iran & Sary and Mohammadi, 2012 \\
\hline & Liver & NA & NA & NA & NA & Khuzestan, Iran & Sary and Mohammadi, 2012 \\
\hline Mudskipper (Gobius boddarti) & Muscle & $7.23 \pm 1.10$ & NA & NA & NA & Dublar Char, Bangladesh & Ahmed et al., 2011 \\
\hline Mudskipper (Periophthalmus barbarus) & Muscle & NA & NA & NA & NA & Port Harcourt, Nigeria & Nwakanma and Hart, 2013 \\
\hline Malaysian Food Regulation 1985 & & NA & NA & NA & NA & & Malaysian Food Act, 2004 \\
\hline $\begin{array}{l}\text { CODEX STAN 193-1995 (Amendment: } \\
\text { 2015) }\end{array}$ & & NA & NA & NA & NA & & $\begin{array}{l}\text { Codex Alimentarius } \\
\text { Commission [CAC], } 2015\end{array}$ \\
\hline $\begin{array}{l}\text { European Commission (EC) No } \\
\text { 1881/2006 }\end{array}$ & & NA & NA & NA & NA & & European Commission, 2006 \\
\hline $\begin{array}{l}\text { Australia New Zealand Food Standards } \\
\text { Code-Schedule } 19\end{array}$ & & NA & NA & NA & NA & & $\begin{array}{l}\text { Australian Government Federal } \\
\text { Register of Legislation, } 2017\end{array}$ \\
\hline $\begin{array}{l}\text { Hong Kong Cap 132V Food } \\
\text { Adulteration (Metallic contamination) } \\
\text { Regulations }\end{array}$ & & NA & 1 & NA & NA & & HK Law, 2019 \\
\hline $\begin{array}{l}\text { Singapore Food Regulations } 1998 \\
\text { (Revised: 2005) }\end{array}$ & & 20 & NA & NA & NA & & $\begin{array}{l}\text { Singapore Food Regulations, } \\
2005\end{array}$ \\
\hline
\end{tabular}


TABLE 3 | Continued

\begin{tabular}{|c|c|c|c|c|c|c|c|}
\hline Biota & Tissues & $\begin{array}{c}\mathrm{Pb} \\
\text { Mean } \pm S D\end{array}$ & $\begin{array}{c}\mathrm{Zn} \\
\text { Mean } \pm S D\end{array}$ & $\begin{array}{c}\text { As } \\
\text { Mean } \pm S D\end{array}$ & $\begin{array}{c}\text { Cd } \\
\text { Mean } \pm S D\end{array}$ & Location & References \\
\hline \multirow{5}{*}{$\begin{array}{l}\text { Giant mudskipper (Periophthalmodon } \\
\text { schlosseri) }\end{array}$} & Muscle & $0.09 \pm 0.03$ & $36.44 \pm 0.22$ & $13.78 \pm 0.10$ & $0.02 \pm 0.00$ & West Coast Peninsular Malaysia & Present study \\
\hline & Gastrointestinal tract & $1.43 \pm 0.02$ & $52.60 \pm 0.33$ & $10.32 \pm 0.01$ & $0.09 \pm 0.00$ & West Coast Peninsular Malaysia & Present study \\
\hline & Gill & $0.96 \pm 0.01$ & $61.04 \pm 1.44$ & $12.10 \pm 0.53$ & $0.05 \pm 0.00$ & West Coast Peninsular Malaysia & Present study \\
\hline & Liver & $0.16 \pm 0.00$ & $25.39 \pm 0.72$ & $7.46 \pm 0.03$ & $0.16 \pm 0.00$ & West Coast Peninsular Malaysia & Present study \\
\hline & Scale & $0.77 \pm 0.00$ & $51.38 \pm 2.09$ & $13.63 \pm 0.28$ & $0.02 \pm 0.00$ & West Coast Peninsular Malaysia & Present study \\
\hline \multirow{3}{*}{$\begin{array}{l}\text { Giant mudskipper (Periophthalmodon } \\
\text { schlosseri) }\end{array}$} & Muscle & $3.20 \pm 0.10$ & $27.80 \pm 1.30$ & NA & $0.40 \pm 0.01$ & Morib, Malaysia & Ikram et al., 2010 \\
\hline & Gill & $5.70 \pm 0.20$ & $36.30 \pm 1.40$ & NA & $0.70 \pm 0.02$ & Morib, Malaysia & Ikram et al., 2010 \\
\hline & Liver & $2.80 \pm 0.20$ & $36.30 \pm 2.70$ & NA & $0.50 \pm 0.03$ & Morib, Malaysia & Ikram et al., 2010 \\
\hline \multirow{3}{*}{$\begin{array}{l}\text { Giant mudskipper (Periophthalmodon } \\
\text { schlosseri) }\end{array}$} & Muscle & $3.90 \pm 0.04$ & $16.00 \pm 1.00$ & NA & $0.40 \pm 0.02$ & Remis, Malaysia & Ikram et al., 2010 \\
\hline & Gill & $6.80 \pm 0.50$ & $28.60 \pm 1.70$ & NA & $0.70 \pm 0.10$ & Remis, Malaysia & Ikram et al., 2010 \\
\hline & Liver & $3.20 \pm 0.30$ & $30.60 \pm 1.30$ & NA & $0.70 \pm 0.01$ & Remis, Malaysia & Ikram et al., 2010 \\
\hline \multirow{5}{*}{$\begin{array}{l}\text { Giant mudskipper (Periophthalmodon } \\
\text { schlosseri) }\end{array}$} & Muscle & $2.09 \pm 1.17^{\mathrm{a}}$ & $44.08 \pm 4.93^{\mathrm{a}}$ & NA & $0.20 \pm 0.11^{a}$ & Sg. Puluh, Klang, Malaysia & Buhari and Ismail T. R., 2017 \\
\hline & Intestine & $10.49 \pm 2.30^{\mathrm{a}}$ & $80.75 \pm 0.72^{\mathrm{a}}$ & NA & $0.08 \pm 0.06^{a}$ & Sg. Puluh, Klang, Malaysia & Buhari and Ismail T. R., 2017 \\
\hline & Gill & $12.09 \pm 0.64^{a}$ & $95.03 \pm 3.50^{\mathrm{a}}$ & NA & $0.28 \pm 0.18^{a}$ & Sg. Puluh, Klang, Malaysia & Buhari and Ismail T. R., 2017 \\
\hline & Liver & $7.90 \pm 0.90^{\mathrm{a}}$ & $34.31 \pm 2.11^{\mathrm{a}}$ & NA & $0.40 \pm 0.20^{a}$ & Sg. Puluh, Klang, Malaysia & Buhari and Ismail T. R., 2017 \\
\hline & Scale & $19.14 \pm 0.38^{a}$ & $96.65 \pm 1.75^{\mathrm{a}}$ & NA & $0.49 \pm 0.20^{\mathrm{a}}$ & Sg. Puluh, Klang, Malaysia & Buhari and Ismail T. R., 2017 \\
\hline Mudskipper (Periophthalmus waltoni) & Muscle & $2.33-12.50$ & $61.94-263.88$ & NA & NA & Hormuz Strait, Persian Gulf & Sarhadizadeh et al., 2013 \\
\hline \multirow[t]{2}{*}{ Mudskipper (Periophthalmus waltoni) } & Muscle & NA & NA & NA & NA & Khuzestan, Iran & Sary and Mohammadi, 2012 \\
\hline & Liver & NA & NA & NA & NA & Khuzestan, Iran & Sary and Mohammadi, 2012 \\
\hline Mudskipper (Gobius boddarti) & Muscle & $4.99 \pm 0.82$ & $98.50 \pm 6.49$ & NA & $0.73 \pm 0.21$ & Dublar Char, Bangladesh & Ahmed et al., 2011 \\
\hline Mudskipper (Periophthalmus barbarus) & Muscle & NA & NA & NA & NA & Port Harcourt, Nigeria & Nwakanma and Hart, 2013 \\
\hline Malaysian Food Regulation 1985 & & 1 & NA & $1^{\#}$ & 1 & & Malaysian Food Act, 2004 \\
\hline $\begin{array}{l}\text { CODEX STAN 193-1995 (Amendment: } \\
\text { 2015) }\end{array}$ & & 0.3 & NA & NA & NA & & $\begin{array}{l}\text { Codex Alimentarius } \\
\text { Commission [CAC], } 2015\end{array}$ \\
\hline $\begin{array}{l}\text { European Commission (EC) No } \\
\text { 1881/2006 }\end{array}$ & & 0.3 & NA & NA & 0.05 & & European Commission, 2006 \\
\hline $\begin{array}{l}\text { Australia New Zealand Food Standards } \\
\text { Code-Schedule } 19\end{array}$ & & 0.5 & NA & $2^{\#}$ & NA & & $\begin{array}{l}\text { Australian Government Federal } \\
\text { Register of Legislation, } 2017\end{array}$ \\
\hline $\begin{array}{l}\text { Hong Kong Cap 132V Food } \\
\text { Adulteration (Metallic contamination) } \\
\text { Regulations }\end{array}$ & & 0.3 & NA & $0.1^{\#}$ & 0.1 & & HK Law, 2019 \\
\hline $\begin{array}{l}\text { Singapore Food Regulations } 1998 \\
\text { (Revised: 2005) }\end{array}$ & & 2 & NA & 1 & NA & & $\begin{array}{l}\text { Singapore Food Regulations, } \\
2005\end{array}$ \\
\hline
\end{tabular}

SD, standard deviation; NA, not available; ${ }^{a}$ mean \pm standard error; " expressed as inorganic arsenic. 
maximum allowable daily fish consumption $\left(\mathrm{CR}_{\mathrm{lim}}\right.$, in $\mathrm{kg} /$ day $)$ for non-carcinogenic elements and carcinogenic elements was calculated based on Equation 5 and Equation 6, respectively (USEPA, 2000).

$$
\begin{aligned}
C R_{\lim } & =\frac{R f D \times B W}{C_{\mathrm{m}}} \\
C R_{\lim } & =\frac{A R L \times B W}{C S F \times C_{\mathrm{m}}}
\end{aligned}
$$

where ARL denotes for maximum acceptable individual lifetime risk $\left(10^{-5}\right)$.

\section{Statistical Analysis}

The data were statistically analyzed using SPSS version 20. The normality of the data was checked by the Shapiro-Wilk test. The exploratory analysis showed that the data deviated from the normal distribution. Thus, a non-parametric Kruskal-Wallis test was used to test for the differences between elemental concentrations in different tissues of the fish. A probability of less than 0.05 was taken as the significant level $(p<0.05)$.

\section{RESULTS AND DISCUSSION}

The elemental concentrations in tissues (muscle, gastrointestinal tract, gill, liver, and scale) of giant mudskippers collected from the sampling sites are shown in Figures $\mathbf{2 A - L}$. The tissues of giant mudskippers collected from this study found that there are differences in the capacities of various tissues to accumulate elements (Figure 2). In this study, fish scale was found to be an important reservoir for elements, such as $\mathrm{Fe}, \mathrm{Ba}, \mathrm{Co}, \mathrm{Mn}$, and Ni. Relatively high concentrations of Fe $(1,641.91 \pm 96.77 \mathrm{mg} / \mathrm{kg}$ $\mathrm{dw}), \mathrm{Ba}(7.07 \pm 0.05 \mathrm{mg} / \mathrm{kg} \mathrm{dw}), \mathrm{Co}(0.32 \pm 0.00 \mathrm{mg} / \mathrm{kg} \mathrm{dw}), \mathrm{Mn}$ $(36.08 \pm 0.04 \mathrm{mg} / \mathrm{kg} \mathrm{dw})$, and $\mathrm{Ni}(4.43 \pm 0.06 \mathrm{mg} / \mathrm{kg} \mathrm{dw})$ were found in the scale of giant mudskippers. Principally, absorption of metals can occur via two pathways: absorption from food through the digestive tract and water and non-dietary exposure through permeable membranes, such as gills and muscles (Crafford and Avenant-Oldewage, 2010; Rajeshkumar and Li, 2018). During exposure, biological barriers, such as the scale, gill, and gut wall, play important roles in controlling the adsorption and absorption of metals prior to transportation to the targeted organs via the circulating system. Fish scale is often regarded as a potential non-lethal indicator of water contamination (Vaid and Hundal, 2019). Being external to the fish body, fish scales are constantly exposed to the surrounding environment and could uptake waterborne elements via dermal exposure. In a comparison work, the fish scales of giant mudskippers collected by Buhari and Ismail T. (2017) were found to accumulate high concentrations of $\mathrm{Pb}, \mathrm{Zn}$, and $\mathrm{Cd}$ compared with other fish tissues. The discrepancy in elemental concentration of fish scales might be due to several factors, such as geographical variation, elemental concentration in the environment, exposure duration, salinity, and temperature (Vaid and Hundal, 2019).

Meanwhile, elements, such as $\mathrm{Cu}(11.04 \pm 0.10 \mathrm{mg} / \mathrm{kg} \mathrm{dw})$, $\mathrm{Pb}(1.35-1.46 \mathrm{mg} / \mathrm{kg} \mathrm{dw})$, and $\mathrm{Al}(726.94 \pm 5.42 \mathrm{mg} / \mathrm{kg} \mathrm{dw})$,

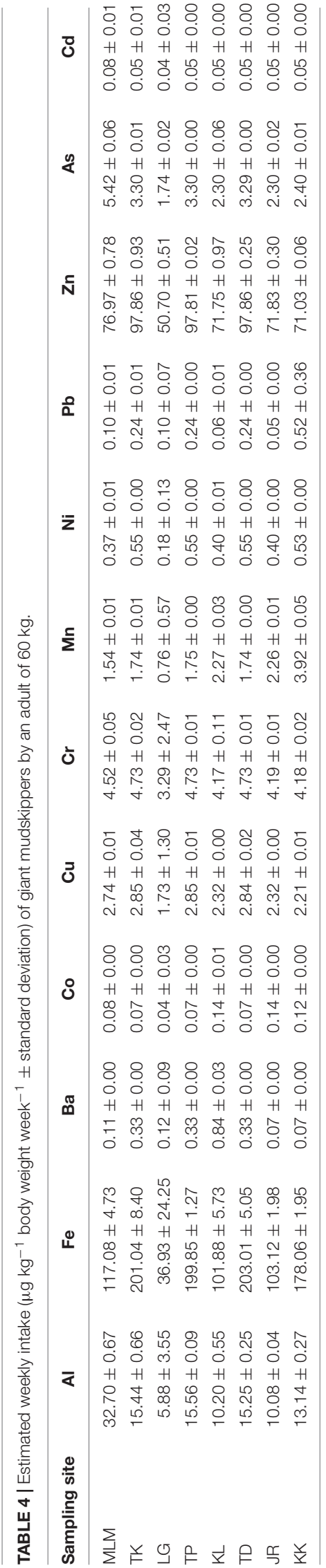


TABLE 5 | Target hazard quotient (THQ) of elements via the consumption of giant mudskippers collected from the West Coast of Peninsular Malaysia.

\begin{tabular}{|c|c|c|c|c|c|c|}
\hline Sampling site & Al & $\mathrm{Fe}$ & $\mathrm{Ba}$ & Co & $\mathrm{Cu}$ & $\mathrm{Cr}$ \\
\hline MLM & $0.005 \pm 0.000$ & $0.024 \pm 0.001$ & $0.000 \pm 0.000$ & $0.039 \pm 0.001$ & $0.010 \pm 0.000$ & $0.215 \pm 0.002$ \\
\hline TK & $0.002 \pm 0.000$ & $0.041 \pm 0.002$ & $0.000 \pm 0.000$ & $0.033 \pm 0.000$ & $0.010 \pm 0.000$ & $0.225 \pm 0.001$ \\
\hline LG & $0.001 \pm 0.001$ & $0.008 \pm 0.005$ & $0.000 \pm 0.000$ & $0.018 \pm 0.013$ & $0.006 \pm 0.005$ & $0.157 \pm 0.118$ \\
\hline TP & $0.002 \pm 0.000$ & $0.041 \pm 0.000$ & $0.000 \pm 0.000$ & $0.033 \pm 0.000$ & $0.010 \pm 0.000$ & $0.225 \pm 0.000$ \\
\hline $\mathrm{KL}$ & $0.001 \pm 0.000$ & $0.021 \pm 0.001$ & $0.001 \pm 0.000$ & $0.065 \pm 0.002$ & $0.008 \pm 0.000$ & $0.198 \pm 0.005$ \\
\hline TD & $0.002 \pm 0.000$ & $0.041 \pm 0.001$ & $0.000 \pm 0.000$ & $0.033 \pm 0.000$ & $0.010 \pm 0.000$ & $0.225 \pm 0.000$ \\
\hline$J R$ & $0.001 \pm 0.000$ & $0.021 \pm 0.000$ & $0.000 \pm 0.000$ & $0.066 \pm 0.001$ & $0.008 \pm 0.000$ & $0.200 \pm 0.000$ \\
\hline KK & $0.002 \pm 0.000$ & $0.036 \pm 0.000$ & $0.000 \pm 0.000$ & $0.059 \pm 0.000$ & $0.008 \pm 0.000$ & $0.199 \pm 0.001$ \\
\hline Sampling site & Mn & $\mathrm{Ni}$ & $\mathrm{Pb}$ & Zn & As & Cd \\
\hline MLM & $0.002 \pm 0.000$ & $0.003 \pm 0.000$ & $0.004 \pm 0.000$ & $0.037 \pm 0.000$ & $2.580 \pm 0.028$ & $0.038 \pm 0.004$ \\
\hline $\mathrm{TK}$ & $0.002 \pm 0.000$ & $0.004 \pm 0.000$ & $0.010 \pm 0.000$ & $0.047 \pm 0.000$ & $1.569 \pm 0.005$ & $0.025 \pm 0.003$ \\
\hline$L G$ & $0.001 \pm 0.001$ & $0.001 \pm 0.001$ & $0.004 \pm 0.003$ & $0.024 \pm 0.000$ & $0.830 \pm 0.008$ & $0.017 \pm 0.013$ \\
\hline TP & $0.002 \pm 0.000$ & $0.004 \pm 0.000$ & $0.010 \pm 0.000$ & $0.047 \pm 0.000$ & $1.569 \pm 0.002$ & $0.025 \pm 0.000$ \\
\hline $\mathrm{KL}$ & $0.002 \pm 0.000$ & $0.003 \pm 0.000$ & $0.002 \pm 0.000$ & $0.034 \pm 0.000$ & $1.094 \pm 0.027$ & $0.025 \pm 0.001$ \\
\hline TD & $0.002 \pm 0.000$ & $0.004 \pm 0.000$ & $0.010 \pm 0.000$ & $0.047 \pm 0.000$ & $1.569 \pm 0.001$ & $0.025 \pm 0.000$ \\
\hline$J R$ & $0.002 \pm 0.000$ & $0.003 \pm 0.000$ & $0.002 \pm 0.000$ & $0.034 \pm 0.000$ & $1.097 \pm 0.009$ & $0.025 \pm 0.000$ \\
\hline KK & $0.004 \pm 0.000$ & $0.004 \pm 0.000$ & $0.021 \pm 0.015$ & $0.034 \pm 0.000$ & $1.145 \pm 0.003$ & $0.022 \pm 0.000$ \\
\hline
\end{tabular}

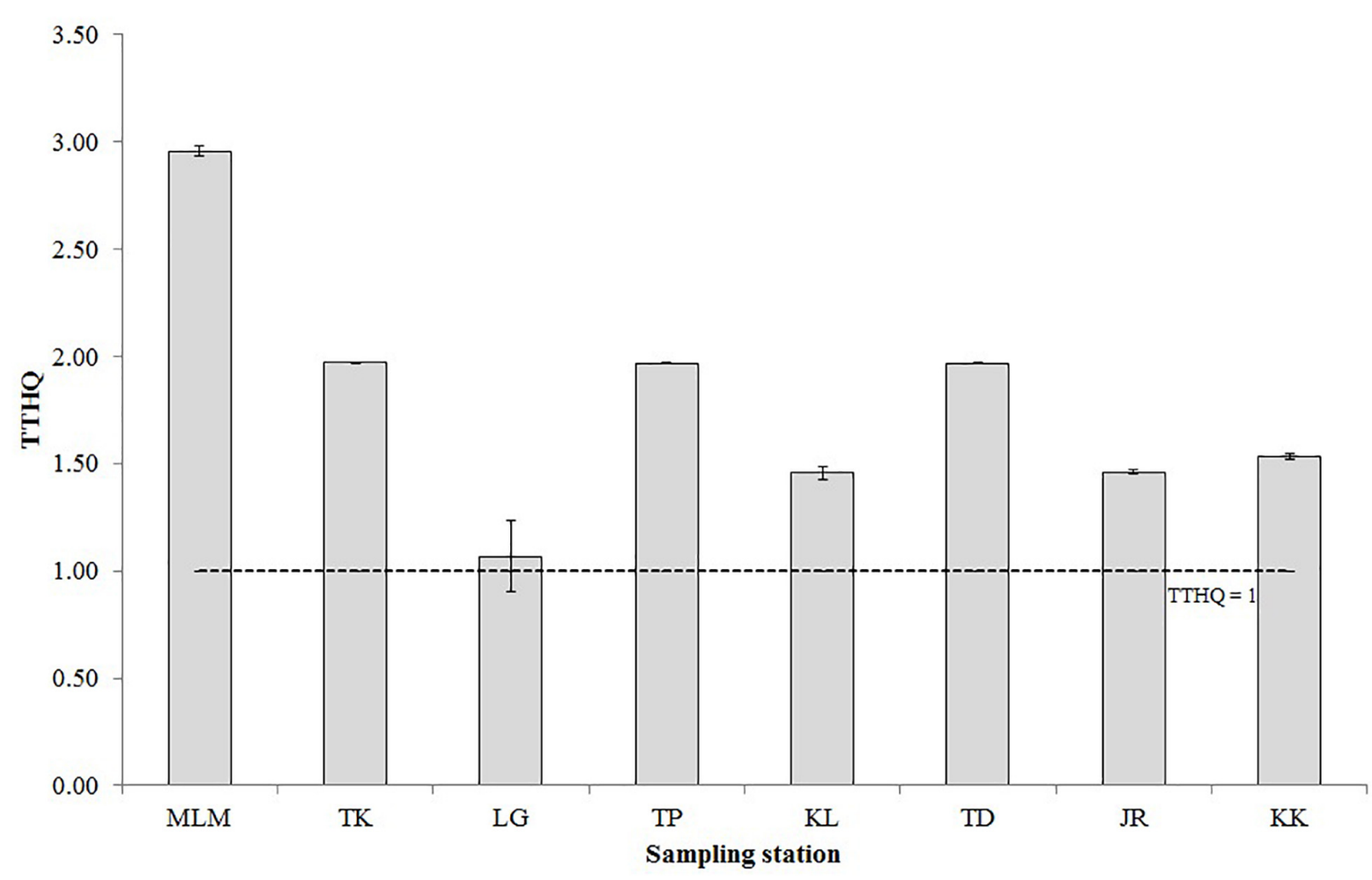

FIGURE 3 | The total target hazard quotient (TTHQ) of elements via the consumption of giant mudskippers. The dotted line marks the $\Pi \mathrm{THQ}$ value of 1 . $T \mathrm{THQ}<1$ indicates that the population is not expected to experience non-cancer risks; $T H Q>1$ indicates that further evaluation is recommended.

were found to accumulate most in the gastrointestinal tract, whereas $\mathrm{Zn}(61.04 \pm 1.44 \mathrm{mg} / \mathrm{kg} \mathrm{dw})$ was found to accumulate mainly in the gill tissue. $\mathrm{Cu}, \mathrm{Pb}$, and $\mathrm{Al}$ concentrations in the gastrointestinal tract probably reflected the accumulation of these elements via food and trophic route, whereas the gill barrier is a good indicator of $\mathrm{Zn}$ exposure through direct contact. The high concentration of $\mathrm{Zn}$ in the current observation could be explained by the large interface provided by the adapted gill membrane of giant mudskippers to store metals from the surrounding atmosphere and water during 
the respiration process. According to Buhari and Ismail T. R. (2017), gills are responsible for $\mathrm{Zn}$ regulation and site of transient metal accumulation, whereby the absorbed metals are distributed and accumulated in the fish body. On the other hand, the concentration of Cd $(0.16 \pm 0.00 \mathrm{mg} / \mathrm{kg} \mathrm{dw})$ was recorded the highest in the liver of giant mudskippers. The liver is an important organ for fishes where diverse metabolic processes and enzyme-catalyzed reactions took place. It also played a vital role in the detoxification of metals (Hajeb et al., 2010). The high concentration of Cd observed in the liver tissue of giant mudskippers implied that this organ had the tendency to metabolize and accumulate Cd from the environment.

A comparative study found that the elemental concentrations $(\mathrm{Cu}, \mathrm{Zn}, \mathrm{Pb}, \mathrm{Cd}$, and $\mathrm{Ni}$ ) from the present study are relatively low compared with the study carried out by Buhari and Ismail T. R. (2017) in investigating the elemental concentrations of giant mudskippers from the West Coast of Peninsular Malaysia. A biomonitoring study by Ikram et al. (2010) along the West Coast of Malaysia also found higher concentrations of $\mathrm{Pb}$ and $\mathrm{Cd}$ in the muscle, gill, and liver tissues of the same fish species. The observed high metal concentrations in their studies are believed to be derived from various anthropogenic activities of the surrounding areas, such as Remis and Sungai Puluh, Klang. In addition, the elemental concentrations ( $\mathrm{Fe}, \mathrm{Ba}, \mathrm{Cu}, \mathrm{Pb}, \mathrm{Zn}$, and $\mathrm{Cd}$ ) in the giant mudskippers collected from this study were lower than those in other species of mudskippers from other geographical regions, such as Bangladesh, Iran, Nigeria, and the Persian Gulf (Table 3). Generally, the mean elemental concentrations in the muscle tissues of the giant mudskippers were below the international and national maximum permissible limits (Table 3), except for $\mathrm{Cr}(1.98 \pm 0.03 \mathrm{mg} / \mathrm{kg})$ and As $(13.78 \pm 0.10 \mathrm{mg} / \mathrm{kg})$. The mean concentration of $\mathrm{Cr}$ in the muscle of giant mudskippers has exceeded the limit $(1.00 \mathrm{mg} / \mathrm{kg})$ stipulated by the Hong Kong Cap 132V Food Adulteration (Metallic contamination) Regulations, whereas the mean concentration of As in the muscle of giant mudskippers has exceeded the $1.00 \mathrm{mg} / \mathrm{kg}$ limit stipulated by the Malaysian Food Regulation 1985 and the Singapore Food Regulations 1998 (Revised: 2005) for inorganic arsenic.

The EWIs of metals due to consumption of giant mudskippers are shown in Table 4. The mean EWI of metals is in the following order: $\mathrm{Fe}>\mathrm{Zn}>\mathrm{Al}>\mathrm{Cr}>\mathrm{As}>\mathrm{Cu}>\mathrm{Mn}>\mathrm{Ni}>\mathrm{Ba}>\mathrm{Pb}>\mathrm{Co}>\mathrm{Cd}$. The highest EWI through the consumption of giant mudskippers for $\mathrm{Al}, \mathrm{As}$, and $\mathrm{Cd}$ was from Merlimau; for $\mathrm{Fe}, \mathrm{Ni}$, and $\mathrm{Zn}$ was from Tanjung Dawai; for Ba was from Klang-Langat estuary; for $\mathrm{Co}$ and $\mathrm{Cr}$ was from Tanjung Kupang; for $\mathrm{Cu}$ and $\mathrm{Pb}$ was from Tanjung Perpat Pasir; and for Mn was from Kuala Kedah. The EWIs were compared with the PTWI as suggested by the JECFA. Considering that the established PTWI is only available for $\mathrm{Al}$ ( $2 \mathrm{mg} \mathrm{kg}^{-1}$ body weight week $\left.{ }^{-1}\right)$, provisional maximum tolerable daily intake (PMTDI) for Fe $\left(0.8 \mathrm{mg} \mathrm{kg}^{-1}\right.$ body weight day $\left.{ }^{-1}\right), \mathrm{Cu}\left(0.5 \mathrm{mg} \mathrm{kg}{ }^{-1}\right.$ body weight $\left.\mathrm{day}^{-1}\right)$, and $\mathrm{Zn}\left(0.3-1.0 \mathrm{mg} \mathrm{kg}^{-1}\right.$ body weight day $\left.{ }^{-1}\right)$, and provisional tolerable monthly intake (PTMI) for Cd $\left(25 \mu \mathrm{g} \mathrm{kg}^{-1}\right.$ body weight month ${ }^{-1}$ ), the comparison of estimated intakes from the current study was made based on these elements. The EWI of Al (0.006$0.033 \mathrm{mg} \mathrm{kg} \mathrm{kg}^{-1}$ body weight week ${ }^{-1}$ ) for the consumption of muscle in giant mudskippers was remarkably below the PTWI as suggested by the JECFA (Food and Agriculture Organisation/World Health Organization [FAO/WHO], 2011). Similarly, the estimated daily intake (EDI) for Fe $(0.04-0.20 \mathrm{mg}$ $\mathrm{kg}^{-1}$ body weight day $\left.{ }^{-1}\right), \mathrm{Cu}\left(0.002-0.003 \mathrm{mg} \mathrm{kg}^{-1}\right.$ body weight day $\left.^{-1}\right)$, and $\mathrm{Zn}\left(0.05-0.10 \mathrm{mg} \mathrm{kg}^{-1}\right.$ body weight day $\left.{ }^{-1}\right)$ was below the FAO/WHO PMTDI, and Cd (0.16-0.34 $\mu \mathrm{g} \mathrm{kg}^{-1}$ body weight month $^{-1}$ ) was below the FAO/WHO PTMI as suggested by the JECFA (Food and Agriculture Organisation/World Health Organization [FAO/WHO], 2019). Based on these estimations, the average consumption of giant mudskippers was unlikely to cause $\mathrm{Al}, \mathrm{Fe}, \mathrm{Cu}, \mathrm{Zn}$, and $\mathrm{Cd}$ intoxication.

The THQ and TTHQ of giant mudskippers from the West Coast of Peninsular Malaysia for individuals who consume the fish every day throughout the year are shown in Table 5 and Figure 3, respectively. The mean THQ for giant mudskippers is in the following order: $\mathrm{As}>\mathrm{Cr}>\mathrm{Co}>\mathrm{Zn}>\mathrm{Fe}>\mathrm{Cd}>\mathrm{Cu}>\mathrm{Pb}>\mathrm{Ni}>\mathrm{Al}>\mathrm{Mn}>\mathrm{Ba}$. The THQ values for all elements were below 1 except for As.

TABLE 6 | Target cancer risk (TR) of elements via the consumption of giant mudskippers collected from the West Coast of Peninsular Malaysia.

\begin{tabular}{lccc}
\hline Sampling site & Cr & $\mathbf{P b}$ & As \\
\hline MLM & $3.23 \times 10^{-4} \pm$ & $1.23 \times 10^{-7} \pm$ & $1.16 \times 10^{-2} \pm$ \\
& $3.56 \times 10^{-6}$ & $6.18 \times 10^{-9}$ & $1.27 \times 10^{-4}$ \\
TK & $3.38 \times 10^{-4} \pm$ & $2.93 \times 10^{-7} \pm$ & $7.06 \times 10^{--3} \pm$ \\
& $1.72 \times 10^{-6}$ & $9.97 \times 10^{-9}$ & $2.06 \times 10^{-5}$ \\
LG & $2.35 \times 10^{-4} \pm$ & $1.17 \times 10^{-7} \pm$ & $3.73 \times 10^{-3} \pm$ \\
& $1.76 \times 10^{-4}$ & $8.69 \times 10^{-8}$ & $3.74 \times 10^{-5}$ \\
TP & $3.38 \times 10^{-4} \pm$ & $2.95 \times 10^{-7} \pm$ & $7.06 \times 10^{-3} \pm$ \\
& $3.81 \times 10^{-7}$ & $5.39 \times 10^{-9}$ & $9.80 \times 10^{-6}$ \\
$\mathrm{KL}$ & $2.98 \times 10^{-4} \pm$ & $6.86 \times 10^{-8} \pm$ & $4.92 \times 10^{-3} \pm$ \\
& $7.61 \times 10^{-6}$ & $1.07 \times 10^{-8}$ & $1.20 \times 10^{-4}$ \\
$\mathrm{TD}$ & $3.38 \times 10^{-4} \pm$ & $2.95 \times 10^{-7} \pm$ & $7.06 \times 10^{-3} \pm$ \\
& $6.46 \times 10^{-7}$ & $8.08 \times 10^{-10}$ & $3.09 \times 10^{-6}$ \\
$\mathrm{JR}$ & $2.99 \times 10^{-4} \pm$ & $6.43 \times 10^{-8} \pm$ & $4.94 \times 10^{-3} \pm$ \\
& $4.44 \times 10^{-7}$ & $1.89 \times 10^{-9}$ & $3.96 \times 10^{-5}$ \\
$\mathrm{KK}$ & $2.98 \times 10^{-4} \pm$ & $6.34 \times 10^{-7} \pm$ & $5.15 \times 10^{-3} \pm$ \\
& $1.19 \times 10^{-6}$ & $4.35 \times 10^{-7}$ & $1.36 \times 10^{-5}$ \\
\hline
\end{tabular}

TABLE 7 | Maximum allowable daily consumption $\left(\mathrm{CR}_{\text {lim }}\right.$, in $\left.\mathrm{g} \mathrm{day}^{-1}\right)$ for giant mudskippers based on non-carcinogenic and carcinogenic effects.

\begin{tabular}{lcccc}
\hline Sampling site & \multicolumn{2}{c}{ Non-carcinogenic } & & \multicolumn{2}{c}{ Carcinogenic } \\
\cline { 2 - 2 } \cline { 5 - 5 } & As & & Cr & As \\
\hline MLM & 1.51 & & 0.12 & 0.10 \\
TK & 2.48 & & 0.12 & 0.17 \\
LG & 6.62 & & 0.17 & 0.44 \\
TP & 2.48 & & 0.12 & 0.17 \\
KL & 3.55 & & 0.13 & 0.24 \\
TD & 2.48 & & 0.12 & 0.17 \\
JR & 3.54 & & 0.13 & 0.24 \\
KK & 3.40 & & 0.13 & 0.23
\end{tabular}


Hence, except for As in giant mudskippers, these findings suggest that the daily elemental exposure via the consumption of giant mudskippers is unlikely to cause any adverse effects. While the As concentration in giant mudskippers might be concerning, the toxic inorganic As concentration in fish generally ranges from 1 to $10 \%$ of total As (Gbogbo et al., 2018). Considering that the calculation of THQ in this study is based on $10 \%$ of total As, the mean TTHQs for all sampling stations were greater than 1 (1.07-2.96), suggesting the possibility of the occurrence of adverse health effects associated with As exposure due to the regular consumption of giant mudskippers collected from the sampling sites.

The TR of $\mathrm{Cr}, \mathrm{Pb}$, and As through the consumption of giant mudskippers is presented in Table 6. The TR values for $\mathrm{Cr}, \mathrm{Pb}$, and As from the consumption of giant mudskippers ranged from $2.35 \times 10^{-4}$ to $3.38 \times 10^{-4}, 6.43 \times 10^{-8} \pm 1.89 \times 10^{-9}$ to $6.34 \times 10^{-7} \pm 4.35 \times 10^{-7}$, and $7.06 \times 10^{-3}$ to $1.16 \times 10^{-2}$, respectively. TR value $1 \times 10^{-6}$ means that there is a chance of developing one cancer case in 1 million individuals. Principally, the calculated TR values of $\mathrm{Cr}$ and As for giant mudskippers at all stations were greater than the acceptable cancer risk of $10^{-5}$ in meal consumption limit (USEPA, 2000), suggesting the possibility of cancer development associated with the consumption of giant mudskippers on a daily basis. Thus, the potential health risks for consumers associated with $\mathrm{Cr}$ and As exposure should receive particular attention.

Owing to the potential health risks, the allowable daily consumption $\left(\mathrm{g} \mathrm{day}^{-1}\right)$ of giant mudskippers is calculated for As and $\mathrm{Cr}$ to determine the amount of fish (in g) that would not be expected to cause adverse health risks. The maximum daily allowable fish consumption of an adult weighing $60 \mathrm{~kg}$ is presented in Table 7. Basically, the intake of 1.51-6.62 g/day of giant mudskipper by an adult of $60 \mathrm{~kg}$ would not be expected to cause As-associated non-carcinogenic effects. Also, the intake of 0.10-0.44 g/day of giant mudskipper collected from sampling sites is not expected to generate As-associated carcinogenic effects. In addition, the consumption of giant mudskippers is advised to be limited to $0.12-0.17 \mathrm{~g} /$ day to prevent developing Cr-associated carcinogenic effects.

\section{CONCLUSION}

The results of the present study provide valuable baseline information concerning concentrations of certain elements, such as $\mathrm{Al}, \mathrm{Fe}, \mathrm{Ba}, \mathrm{Co}, \mathrm{Cr}, \mathrm{Mn}$, and As, their distributions, health risk assessment, and fish consumption limits for giant mudskippers collected from the West Coast of Peninsular Malaysia. A comparative study showed that concentrations of $\mathrm{Cu}, \mathrm{Zn}, \mathrm{Pb}, \mathrm{Cd}$, and $\mathrm{Ni}$ from the present study are relatively low compared with previous studies from the same region. The concentrations of elements, such as $\mathrm{Fe}, \mathrm{Ba}, \mathrm{Co}, \mathrm{Mn}$, and $\mathrm{Ni}$, were found to be higher in the fish scales, and $\mathrm{Cu}, \mathrm{Pb}$, and $\mathrm{Al}$ were found to accumulate most in the gastrointestinal tract of giant mudskippers. $\mathrm{Zn}$ was found to accumulate mainly in the gill tissue, whereas $\mathrm{Cd}$ was found to accumulate most in the liver. Although gastrointestinal, gills, scales, and liver are not consumed by consumers, they could be potential bioindicators of elemental pollution for intertidal environments. Notably, the muscle tissues of giant mudskippers are not heavily burdened with elements as the concentrations are below the permissible limits, except for $\mathrm{Cr}$ and As. The estimated intakes for $\mathrm{Al}$, $\mathrm{Fe}, \mathrm{Cu}, \mathrm{Zn}$, and $\mathrm{Cd}$ were all below the tolerable intakes recommended by the JECFA. TTHQ indicated that long-term consumption of As would cause possible non-carcinogenic risk to the consumers. Meanwhile, TR suggested that daily $\mathrm{Cr}$ and As exposure through the consumption of giant mudskippers would cause potential cancer risk. Thus, periodical monitoring of elements, such as $\mathrm{Cr}$ and As, at hotspot areas using indicator species is recommended. Such monitoring studies could help in deciphering the health status of the intertidal zone and safeguard human lives. Nevertheless, there is a need for future research, whereby speciation and total diet study could be conducted to assess the exact dietary exposure and safety risk.

\section{DATA AVAILABILITY STATEMENT}

The original contributions presented in the study are included in the Supplementary Material, further inquiries can be directed to the corresponding authors.

\section{AUTHOR CONTRIBUTIONS}

LL and AA designed the study. LL carried out field and laboratory analyses. AA and FY supervised and provide resources. All authors contributed to data analysis and writing of the manuscript.

\section{FUNDING}

This research was financially supported by the Universiti Putra Malaysia (GP-IPM/2019/9679100) and Fundamental Research Grant Scheme (FRGS/1/11/STWN/UPM/02/32) from the Ministry of Higher Education Malaysia.

\section{ACKNOWLEDGMENTS}

The authors are very grateful to the reviewer for their constructive comments and suggestion for improving this manuscript. The authors would like to thank Ng Tzu Shan, Lim Wan Ying, Farhah Amalya Ismail, and Lim Ai Phing for their help during field sampling. A special thanks to Dr. Zakiah Ponrahono for her kind assistance in editing the map.

\section{SUPPLEMENTARY MATERIAL}

The Supplementary Material for this article can be found online at: https://www.frontiersin.org/articles/10.3389/fmars.2020. 618284/full\#supplementary-material 


\section{REFERENCES}

Ahmed, A. S. S., Rahman, M., Sultana, S., Babu, S. M. O. F., and Sarker, M. S. I. (2019). Bioaccumulation and heavy metal concentration in tissues of some commercial fishes from the Meghna River Estuary in Bangladesh and human health implications. Mar. Poll. Bull. 145, 436-447. doi: 10.1016/j.marpolbul. 2019.06.035

Ahmed, K., Mehedi, Y., Haque, R., and Mondol, P. (2011). Heavy metal concentrations in some macrobenthic fauna of the Sundarbans mangrove forest, south west coast of Bangladesh. Environ. Monitor. Assess. 177, 505-514. doi: 10.1007/s10661-010-1651-9

Ahmed, M. K., Shaheen, N., Islam, M. S., Habibullah-al-Mamun, M., Islam, S., Mohiduzzaman, M., et al. (2015). Dietary intake of trace elements from highly consumed cultured fish (Labeo rohita, Pangasius pangasius and Oreochromis mossambicus) and human health risk implications in Bangladesh. Chemosphere 128, 284-292. doi: 10.1016/j.chemosphere.2015.02.016

Ali, H., Khan, E., and Ilahi, I. (2019). Environmental chemistry and ecotoxicology of hazardous heavy metals: environmental persistence, toxicity, and bioaccumulation. J. Chem. 2019:6730305. doi: 10.1155/2019/6730305

Aris, A. Z., and Looi, L. J. (2015). "Estuaries ecosystems health status - profiling the advancements in metal analysis," in Environmental Management and Governance: Advances in Coastal and Marine Resources, eds C. W. Finkl and C. Makowski (Berlin: Springer International Publishing), 429-453. doi: 10.1007/ 978-3-319-06305-8_16

Australian Government Federal Register of Legislation (2017). Australia New Zealand Food Standards Code - Schedule 19 - Maximum Levels of Contaminants and Natural Toxicants. Available online at: https://www.legislation.gov.au/Details/F2017C00333 (accessed October 13, 2020).

Buhari, T. R., and Ismail, A. (2016). Correlations between Geo-Chemical speciation of heavy metals $(\mathrm{Cu}, \mathrm{Zn}, \mathrm{Pb}, \mathrm{Cd}$ and $\mathrm{Ni}$ ) in surface sediments and their concentrations in giant mudskipper (Periophthalmodon schlosseri) collected from the west coast of peninsular Malaysia. J. Geosci. Environ. Protect. 4, 28-36. doi: 10.4236/gep.2016.41003

Buhari, T., and Ismail, A. (2017). Evaluation of metallothionein content in giant mudskipper Periophthalmodon schlosseri collected from west coast of peninsular Malaysia to assess environmental metal pollution. J. Coast. Life Med. 5, 279-287.

Buhari, T. R., and Ismail, A. (2017). Bio monitoring of heavy metals (Cu, $\mathrm{Zn}, \mathrm{Pb}, \mathrm{Cd}$ and $\mathrm{Ni}$ ) in the West Coast of Peninsular Malaysia using giant mudskipper Periophthalmodon schlosseri (Pallas 1770). J. Life Sci. Biomed. 7, 90-109.

Codex Alimentarius Commission [CAC] (2015). General Standard for Contaminants and Toxins in Food and Feed (CODEX STAN 193-1995) (Amended 2015). Rome: Joint FAO/WHO Food Standards Programme, FAO.

Crafford, D., and Avenant-Oldewage, A. (2010). Bioaccumulation of non-essential trace metals in tissues and organs of Clarias gariepinus (sharptooth catfish) from the Vaal River system: strontium, aluminium, lead and nickel. Water SA 36, 621-640.

European Commission (2006). Setting maximum levels for certain contaminants in foodstuffs. consolidated version of the regulation (EC) No 1881/2006 of 19 December 2006. Off. J. EU, L 364, 5-24.

Food and Agriculture Organisation/World Health Organization [FAO/WHO] (2011). JECFA Aluminium Evaluations. Available online at: https://apps.who. int/food-additives-contaminants-jecfa-database/chemical.aspx? chemID $=298$ (accessed October, 2020).

Food and Agriculture Organisation/World Health Organization [FAO/WHO] (2019). JECFA List of Chemicals in Functional Category Food Contaminant. Available online at: https://apps.who.int/food-additives-contaminants-jecfadatabase/search.aspx?fcc $=2$ (accessed October 12, 2020).

Gbogbo, F., Arthur-Yartel, A., Bondzie, J. A., Dorleku, W.-P., Dadzie, S., Kwansa-Bentum, B., et al. (2018). Risk of heavy metal ingestion from the consumption of two commercially valuable species of fish from the fresh and coastal waters of Ghana. PLoS One 13:e0194682. doi: 10.1371/journal.pone.019 4682

GEMS/WHO (2012). Global Environment Monitoring System (GEMS)/Food Cluster Diets 2012. Available online at: http://www.who.int/nutrition/ landscape_analysis/nlis_gem_food/en/ (accessed June 01, 2016).
Gu, Y.-G. (2018). Heavy metal fractionation and ecological risk implications in the intertidal surface sediments of Zhelin Bay. South China. Mar. Poll. Bull. 129, 905-912. doi: 10.1016/j.marpolbul.2017.10.047

Hajeb, P., Jinap, S., and Ahmad, I. (2010). Biomagnifications of mercury and methylmercury in tuna and mackerel. Environ. Monitor. Assess. 171, 205-217. doi: 10.1007/s10661-009-1272-3

Haris, H., and Aris, A. Z. (2015). Distribution of metals and quality of intertidal surface sediment near commercial ports and estuaries of urbanized rivers in Port Klang, Malaysia. Environ. Earth Sci. 73, 7205-7218. doi: 10.1007/s12665014-3900-7

HK Law (2019). Laws of Hong Kong 1983. Food Adulteration (Metallic Contamination) (Amendment) Regulation 2018 [CAP. 132, section 55(1)] Available online at: https://www.elegislation.gov.hk/hk/cap132V@2019-0919T00:00:00 (accessed December 14, 2020).

Hossen, M. F., Hamdan, S., and Rahman, M. R. (2015). Review on the risk assessment of heavy metals in malaysian clams. Sci. World J. 2015:7. doi: 10. $1155 / 2015 / 905497$

Hwang, D.-W., Kim, P.-J., Kim, S.-G., Sun, C.-I., Koh, B.-S., Ryu, S.-O., et al. (2019). Spatial distribution and pollution assessment of metals in intertidal sediments, Korea. Environ. Sci. Poll. Res. 26, 19379-19388. doi: 10.1007/s11356019-05177-z

Ikram, M. M., Ismail, A., Yap, C. K., and Azwady, A. A. N. (2010). Levels of heavy metals $(\mathrm{Zn}, \mathrm{Cu}, \mathrm{Cd}$, and $\mathrm{Pb}$ ) in mudskippers (Periophthalmodon schlosseri) and sediments collected from intertidal areas at Morib and Remis, Peninsular Malaysia. Toxicol. Environ. Chem. 92, 1471-1486. doi: 10.1080/ 02772241003614304

Khaironizam, M., and Norma-Rashid, Y. (2005). "Distribution of mudskippers (Gobiidae: Oxudercinae) on the Selangor coast," in Ecology of Klang Strait. Faculty of Science, eds A. Sasekumar and V. Chong (Kuala Lumpur: University of Malaya press).

Khatri, N., and Tyagi, S. (2015). Influences of natural and anthropogenic factors on surface and groundwater quality in rural and urban areas. Front. Life Sci. 8:23-39. doi: 10.1080/21553769.2014.933716

Liu, J., Cao, L., and Dou, S. (2019). Trophic transfer, biomagnification and risk assessments of four common heavy metals in the food web of Laizhou Bay, the Bohai Sea. Sci. Total Environ. 670, 508-522. doi: 10.1016/j.scitotenv.2019.03. 140

Looi, L. J., Aris, A. Z., Haris, H., Yusoff, F. M., and Hashim, Z. (2016). The levels of mercury, methylmercury and selenium and the selenium health benefit value in grey-eel catfish (Plotosus canius) and giant mudskipper (Periophthalmodon schlosseri) from the Strait of Malacca. Chemosphere 152, 265-273. doi: 10.1016/ j.chemosphere.2016.02.126

Looi, L. J., Aris, A. Z., Yusoff, F. M., Isa, N. M., and Haris, H. (2019). Application of enrichment factor, geoaccumulation index, and ecological risk index in assessing the elemental pollution status of surface sediments. Environ. Geochem. Health 41, 27-42. doi: 10.1007/s10653-018-0149-1

MacKinnon, J. V., Yvonne, I., and Murray, N. (2012). IUCN Situation Analysis on East and Southeast Asian Intertidal Habitats, with Particular Reference to the Yellow Sea (including the Bohai Sea) (Occasional Paper of the IUCN Species Survival Commission No 47. Gland: IUCN.

Madricardo, F., Foglini, F., Campiani, E., Grande, V., Catenacci, E., Petrizzo, A., et al. (2019). Assessing the human footprint on the sea-floor of coastal systems: the case of the Venice Lagoon. Italy. Sci. Rep. 9:6615. doi: 10.1038/s41598-01943027-7

Malaysian Food Act (2004). Food act 1983 (Act 281) and Regulations. Kuala Lumpur: International Law Book Services.

Mottalib, M. A., Zilani, G., Suman, T. I., Ahmed, T., and Islam, S. (2018). Assessment of trace metals in consumer chickens in Bangladesh. J. Health Poll. 8, 181208-181208. doi: 10.5696/2156-9614-8.20.181208

Naccari, C., Cicero, N., Ferrantelli, V., Giangrosso, G., Vella, A., Macaluso, A., et al. (2015). Toxic metals in pelagic, benthic and demersal fish species from mediterranean FAO Zone 37. Bull. Environ. Contaminat. Toxicol. 95, 567-573. doi: 10.1007/s00128-015-1585-6

Ng, Y. H., Mohamed, M., Othman, M. N. A., and Tokiman, L. (2019). Diversity and behaviour of mudskippers of Tanjung Piai, Pontian, Johor. IOP Conf. Series: Earth Environ. Sci. 269:012037.

Nwakanma, C., and Hart, A. I. (2013). Determination of barium levels in soft tissue of niger Delta mudskipper, Periophthalmus barbarous (L) using buck scientific 
atomic absorption and emission spectrophotometer 200A (AAS). Curr. Res. J. Biol. Sci. 5, 195-197.

Omar, M. N., Siti-Fairuz, C. O., Hasan, M. T., Nor-Nazuha, M. N., Nor-Dalilah, M. N., and Kamaruzzaman, Y. (2010). Study on $\omega$-fatty acids from Malaysian giant mudskipper (Periophthalmodon schlosseri) fish oil. Oriental J. Chem. 26, 861-864.

Radojević, M., and Bashkin, V. N. (2006). Practical Environment Analysis, 2nd ed. Cambridge: The Royal Society of Chemistry.

Rajeshkumar, S., and Li, X. (2018). Bioaccumulation of heavy metals in fish species from the Meiliang Bay, Taihu Lake, China. Toxicol. Rep. 5, 288-295. doi: 10. 1016/j.toxrep.2018.01.007

Sany, S. B. T., Tajfard, M., Rezayi, M., Rahman, M. A., and Hashim, R. (2019). "Chapter 20 - the west coast of Peninsular Malaysia," in World Seas: an Environmental Evaluation (Second Edition), ed. C. Sheppard (Cambridge, MA: Academic Press), 437-458. doi: 10.1016/B978-0-08-100853-9.00050-6

Sarhadizadeh, N., Afkhami, M., Ehsanpour, M., and Bastami, K. D. (2013). Heavy metal pollution monitoring in the northern coast of Hormuz Strait (Persian Gulf): plasma enzyme variations in Periophthalmus waltoni. Comp. Clin. Pathol. 23, 1063-1067.

Sary, A. A., and Mohammadi, M. (2012). Mercury concentrations in commercial fish from freshwater and saltwater. Bull. Environ. Contaminat. Toxicol. 88, $162-165$.

Shazili, N. A. M., Yunus, K., Ahmad, A. S., Abdullah, N., and Rashid, M. K. A. (2006). Heavy metal pollution status in the Malaysian aquatic environment. Aquatic Ecosystem Health Manag. 9, 137-145. doi: 10.1080/ 14634980600724023

Singapore Food Regulations (2005). Sale if Food Act (Chapter 283, Section 56 (1)). Incidental Constituents in Food. Available online at: https://sso.agc.gov.sg/SL/ SFA1973-RG1?DocDate=20190830\#legis (accessed October 13, 2020).

Tavakoly Sany, S. B., Hashim, R., Rezayi, M., Salleh, A., and Safari, O. (2014). A review of strategies to monitor water and sediment quality for a sustainability assessment of marine environment. Environ. Sci. Poll. Res. 21, 813-833. doi: 10.1007/s11356-013-2217-5

Thanh-Nho, N., Marchand, C., Strady, E., Vinh, T.-V., and Nhu-Trang, T.T. (2019). Metals geochemistry and ecological risk assessment in a tropical mangrove (Can Gio. Vietnam). Chemosphere 219, 365-382. doi: 10.1016/j. chemosphere.2018.11.163

Trevizani, T. H., Domit, C., Vedolin, M. C., Angeli, J. L. F., and Figueira, R. C. L. (2019). Assessment of metal contamination in fish from estuaries of southern and southeastern Brazil. Environ. Monit. Assess. 191:308. doi: 10.1007/s10661019-7477-1

USEPA (2000). Guidance for Assessing Chemical Contaminant Data for Use in Fish Advisories: Risk Assessment and Fish Consumption Limits, third Edn. Washington, DC: U.S. Environmental Protection Agency (USEPA).

USEPA (2016). Human Health Risk Assessment. Available online at: https: //www.epa.gov/risk/human-health-risk-assessment (accessed December 14, 2020).

USEPA (2020). Integrated Risk Information System (IRIS) summary table. Wachington, DC: United State Environmental Protection Agency (USEPA).

USEPA Method 3051A (2007). Microwave Assisted Acid Digestion of Sediments, Sludges, Soils and Oils, in Test Methods For Evaluating Solid Waste. Washington, DC: US Environmental Protection Agency.

Vaid, V., and Hundal, S. S. (2019). Light microscopic studies to evaluate fish scales as non-invasive indicators of heavy metal-contaminated waters. Environ. Monit. Assess. 191:638. doi: 10.1007/s10661-019-7801-9
Veiga, K., Pedro, C. A., Ferreira, S. M. F., and Gonçalves, S. C. (2019). Monitoring metal pollution on coastal lagoons using Cerastoderma edule-a report from a moderately impacted system in Western Portugal (Óbidos Lagoon). Environ. Sci. Poll. Res. 26, 2710-2721. doi: 10.1007/s11356-018-3705-4

Wei, J., and Cen, K. (2020). Assessment of human health risk based on characteristics of potential toxic elements (PTEs) contents in foods sold in Beijing. China. Sci. Total Environ. 703:134747. doi: 10.1016/j.scitotenv.2019. 134747

Wilkes, R., Bennion, M., McQuaid, N., Beer, C., McCullough-Annett, G., Colhoun, K., et al. (2017). Intertidal seagrass in Ireland: pressures, WFD status and an assessment of trace element contamination in intertidal habitats using Zostera noltei. Ecol. Indicators 82, 117-130. doi: 10.1016/j.ecolind.2017.06.036

Yap, C. K., and Cheng, W. H. (2009). Heavy metal concentrations in Nerita lineata: the potential as a biomonitor for heavy metal bioavailability and contamination in the tropical intertidal area. Mar. Biodivers. Records 2:e46. doi: 10.1017/ S1755267209000505

Yap, C. K., Cheng, W. H., Ismail, A., Ismail, A. R., and Tan, S. G. (2009). Biomonitoring of heavy metal $(\mathrm{Cd}, \mathrm{Cu}, \mathrm{Pb}$, and $\mathrm{Zn})$ concentrations in the west intertidal area of Peninsular Malaysia by using Nerita lineata. Toxicol. Environ. Chem. 91, 29-41. doi: 10.1080/02772240801968706

Yap, C. K., Ismail, A., and Tan, S. G. (2003a). Cd and Zn concentrations in the straits of Malacca and intertidal sediments of the west coast of Peninsular Malaysia. Mar. Poll. Bull. 46, 1349-1353. doi: 10.1016/S0025-326X(03)00193-0

Yap, C. K., Ismail, A., and Tan, S. G. (2003b). Mercury concentrations in the surface sediments of the intertidal area along the west coast of Peninsular Malaysia. Toxicol. Environ. Chem. 85, 13-21. doi: 10.1080/0277224031000135049

Yap, C. K., and Pang, B. H. (2011). Assessment of $\mathrm{Cu}, \mathrm{Pb}$, and $\mathrm{Zn}$ contamination in sediment of north western Peninsular Malaysia by using sediment quality values and different geochemical indices. Environ. Monit. Assess. 183, 23-39. doi: 10.1007/s10661-011-1903-3

You, X., Sun, M., Li, J., Bian, C., Chen, J., Yi, Y., et al. (2018). Mudskippers and their genetic adaptations to an amphibious lifestyle. Animals Open Access J. MDPI 8:24. doi: 10.3390/ani8020024

Zhao, M., Wang, E., Xia, P., Feng, A., Chi, Y., and Sun, Y. (2019). Distribution and pollution assessment of heavy metals in the intertidal zone environments of typical sea areas in China. Mar. Poll. Bull. 138, 397-406. doi: 10.1016/j. marpolbul.2018.11.050

Zhu, H., Bing, H., Yi, H., Wu, Y., and Sun, Z. (2018). Spatial distribution and contamination assessment of heavy metals in surface sediments of the caofeidian adjacent sea after the land reclamation, Bohai Bay. J. Chem. 2018:13. doi: 10.1155/2018/2049353

Zulkifli, S. Z., Mohamat-Yusuff, F., Ismail, A., and Miyazaki, N. (2012). Food preference of the giant mudskipper Periophthalmodon schlosseri (Teleostei : Gobiidae). Knowl. Managt. Aquatic Ecosyst. 405:7. doi: 10.1051/kmae/2012013

Conflict of Interest: The authors declare that the research was conducted in the absence of any commercial or financial relationships that could be construed as a potential conflict of interest.

Copyright (C) 2021 Looi, Aris, Isa, Yusoff and Haris. This is an open-access article distributed under the terms of the Creative Commons Attribution License (CC BY). The use, distribution or reproduction in other forums is permitted, provided the original author(s) and the copyright owner(s) are credited and that the original publication in this journal is cited, in accordance with accepted academic practice. No use, distribution or reproduction is permitted which does not comply with these terms. 